Relations industrielles

Industrial Relations

\title{
Les syndicats ouvriers et la participation aux bénéfices aux États-Unis
}

\section{Labor Attitudes on Profit Sharing in the United States}

\section{Gaston Cholette}

Volume 14, numéro 2, avril 1959

URI : https://id.erudit.org/iderudit/1022315ar

DOI : https://doi.org/10.7202/1022315ar

Aller au sommaire du numéro

Éditeur(s)

Département des relations industrielles de l’Université Laval

ISSN

0034-379X (imprimé)

1703-8138 (numérique)

Découvrir la revue

Citer cet article

Cholette, G. (1959). Les syndicats ouvriers et la participation aux bénéfices aux États-Unis. Relations industrielles / Industrial Relations, 14(2), 200-210.

https://doi.org/10.7202/1022315ar
Résumé de l'article

Dans un précédent numéro, l'auteur a analysé l'état et le développement de ce phénomène. Il expose ici l'attitude des syndicats ouvriers.
Tous droits réservés (C) Département des relations industrielles de l’Université Laval, 1959
Ce document est protégé par la loi sur le droit d'auteur. L'utilisation des services d'Érudit (y compris la reproduction) est assujettie à sa politique d'utilisation que vous pouvez consulter en ligne.

https://apropos.erudit.org/fr/usagers/politique-dutilisation/ 


\section{Les syndicats ouvriers et la participation aux bénéfices aux Etats-Unis}

\section{Gaston Cholette}

Dans un précédent numéro, lauteur a analysé létat et le développement de ce phénomène. Il expose ici l'attitude des syndicats ouvriers.

Tout aperçu sur le mouvement de participation aux bénéfices aux Etats-Unis ou ailleurs serait fort incomplet s'il ne rendait pas compte des attitudes ouvrières. Comme on l'a vu déjà, la participation aux bénéfices a été et demeure encore à l'heure actuelle une initiative patronale et gouvernementale. Les patrons y ont vu un moyen de faire partager leur conception de l'entreprise et de l'économie par leurs ouvriers, tandis que le gouvernement y a vu une formule efficace pour sauvegarder la paix industrielle et consolider le système capitaliste. Les ouvriers, eux, n’ont pas joué un rôle très actif dans le développement de ce mouvement.

On peut étudier les attitudes ouvrières à trois paliers différents: a) au niveau des ouvriers d'une entreprise ou des ouvriers de tout le pays considérés comme des individus; b) au niveau des officiers locaux de syndicats et enfin, c) à l'échelon des unions ouvrières nationales ou internationales. On peut aussi les considérer dans le temps et voir si, chronologiquement, il y a eu stabilité ou évolution.

L'enquête du sous-comité sénatorial en 1938 révèle une attitude favorable des travailleurs, pris individuellement, à l'idée de participation aux bénéfices. Le sous-comité avait fait parvenir par la poste un questionnaire à un certain nombre d'employés de 104 établissements industriels comptant environ 90,000 ouvriers et ne pratiquant pas le partage des profits. La première question demandait aux ouvriers s'ils étaient en faveur du partage des bénéfices. Or, $87 \%$ de ceux qui firent parvenir une réponse se prononcèrent dans l'affirmative. ${ }^{1}$

CHOLETTE, Gaston, M.Sc.Soc. (Laval), Docteur en droit (Paris), conciliateur, Ministère provincial du travail.

(1) Senate Report, Survey of Experiences in Profit Sharing and Possibilities of Incentive Taxation, No. 610, United States Government Printing Office, Washington, 1939, p. 120. 
En 1943, une enquête était lancée par la revue «Fortune 》 pour connaître l'attitude des ouvriers au sujet de la distribution des profits. ${ }^{2}$ $\mathrm{Si}$ une compagnie paie d'excellents gages aux ouvriers et des dividendes raisonnables aux actionnaires, lequel des groupes suivants devrait, demandait-on, recevoir les profits qui restent: les administrateurs, les travailleurs, les actionnaires ou le gouvernement? Voici le pourcentage des réponses pour chaque groupe: les travailleurs, $61.9 \%$; le gouvernement, $40.4 \%$; les actionnaires, $23.6 \%$; les administrateurs, $17.2 \%$; indécis, $7.9 \%$.

Ainsi, plus des $3 / 5$ de ces travailleurs croient que les ouvriers devraient recevoir la totalité ou une partie des profits qui restent après 1a distribution de justes dividendes aux actionnaires. Il va sans dire que ces travailleurs ne sont pas nécessairement pour cela en faveur du système du partage des profits au sens strict, car on peut interpréter tout aussi bien leur réponse comme une condamnation des profits trop élevés ou une réclamation de meilleurs salaires.

En 1948, la «National Industrial Conference Board» publiait le rapport d'une enquête faite auprès de 202 compagnies qui avaient déjà appliqué ou qui appliquaient à ce moment-là le partage des profits. Au cours de la période de 1937 à 1947, 35 de ces plans avaient été abandonnés: dans 7 cas, soit $20.0 \%$ du total, la raison invoquée était l'attitude de l'union ouvrière; dans 3 cas, soit $8.6 \%$ du total, on attribuait l'échec à l'indifférence des travailleurs ou à leur mécontentement; dans 2 cas, soit $5.7 \%$ du total, on disait que les ouvriers préféraient recevoir le montant dans leur enveloppe de paye. ${ }^{3}$

Au niveau des unions locales et de leurs officiers, les attitudes au sujet de la participation aux bénéfices reflètent les tendances opposées, d'une part, des désirs des ouvriers pris individuellement, et, d'autre part, des politiques des unions nationales et internationales. En somme, là où la pression de l'employeur et des travailleurs est suffisamment forte, les dirigeants locaux ne font pas obstacle au partage des profits ei coopèrent même à sa réalisation. Lorsque cette pression locale ne se fait pas sentir suffisamment, ils inclineront plutôt vers une attitude de résistance passive ou active afin de se conformer à la politique des groupements nationaux. Il serait long et de peu d'intérêt de présenter

(2) Fortune Survey, Fortune, février 1943, pp. 8-10.

(3) Cité dans Revised Profit Sharing Manual, op. cit., p. 29. 
ici un inventaire détaillé d'exemples concrets pour confirmer expérimentalement ces affirmations.

Quant aux positions des principaux chefs ouvriers et des grandes centrales ouvrières, on peut plus facilement en faire l'étude car les déclarations sur ce point sont nombreuses et concluantes. En général, le travail organisé aux Etats-Unis a été officiellement et systématiquement opposé à la participation aux bénéfices. Samuel Gompers, le premier président de la Fédération américaine du travail, déclarait en 1899:

"I would look upon such propositions with a very great deal of suspicion. There have been few, if any, of these concerns (companies with Profit Sharing plans) that have been comparatively fair to their employees." 4

Cette attitude d'indifférence, pour ne pas dire de dédain, se transforme par la suite en opposition ouverte au sein du mouvement ouvrier. En 1938, devant le sous-comité sénatorial, John L. Lewis déclarait dans son témoignage:

"Labor's disillusioned experience in regard to profit-sharing plans has been that they have been used as a device to avoid the payment of an immediate decent wage and made labor dependent upon haphazard industrial and financial policies of management...

Labor cannot eat or live on hopes of participation in profitsharing plans..." 5

Au cours de la même enquête sénatoriale, William Green, alors président de la Fédération américaine du travail, disait pour sa part:

"It follows that are were equally unwilling to see the scope of collective bargaining narrowed so that profit sharing or any other new provision affecting work relationships must mean an extension of collective bargaining to the new field. All of the terms and conditions of payment for work should be determined through joint conferences of representatives of management and workers concerned and carried to mutual agreement upon issues discussed." 6

Cette déclaration était en quelque sorte un témoignage en faveur de la négociation collective plutôt qu'une adhésion à l'idée du partage des profits. Elle indique la méfiance ouvrière à l'endroit d'une formule

(4) Cf. Thompson, op. cit., p. 43; aussi Bryce M. Stewart et Walter J. Couper, Profit Sharing and Stock Ownership for Wage Earners and Executives, Monograph No. 10 , p. 6.

(5) Survey of Experiences in Profit Sharing and Possibilities of Incentive Taxation, Hearings before a Subcommittee of the Committee on Finance, U.S. Senate, S. RES. 215, November 21 - December 14, 1938, U.S. Printing Office, Washington, 1939, pp. 189-190.

(6) Idem, pp. 105-106. 
que trop d'employeurs avaient cherché à soustraire à la négociation. Et Green ajoutait:

\begin{abstract}
"After that is established, then if the earnings of the industry will justify an equitable distribution of the profits of industry between investors, management, and employees, let it be done, with a full understanding and in full cooperation with the representatives of the workers. The one trouble about profit sharing, as practiced by a number of corporations, is that it has created suspicion, distrust, because the workers know nothing about the basis upon which the profits were distributed. If it is to be put into effect in a practical and satisfactory way, there is a great need of frankness and open dealing between the management or the corporation and the workers themselves. Let the workers know the truth." ?
\end{abstract}

Tout en ne déclarant pas la guerre aux principes qui sont à la base de la philosophie de la participation aux bénéfices mais plutôt à la façon dont ce système avait toujours fonctionné, Green s'est quand même prononcé très catégoriquement contre toute mesure législative qui aurait pour effet d'accorder des exemptions de taxe aux entreprises qui suivent cette politique. ${ }^{8}$

Les chefs du Congrès des organisations industrielles ont adopté une attitude moins conciliante que celle de William Green. Philip Murray était d'avis que les ouvriers, s'y connaissant peu en comptabilité, considéraient les plans de partage des profits comme des sources de conflit et non comme des instruments de pacification des relations de travail. ${ }^{9}$

L'attaque la plus virulente par une union nationale ouvrière contre les modes de calcul des profits a été faite par les «United Electrical, Radio and Machine Workers of America», en 1943. ${ }^{10}$ Cette union ouvrière soutenait que les moyens de cacher les profits étaient pratiquement illimités et que ces moyens étaient bien connus de la plupart des grosses entreprises. Ainsi, puisqu'il est possible de sauver des montants considérables par des méthodes de comptabilité frauduleuses, les grosses compagnies ne se gênent pas pour débourser les fortes sommes exigées par les comptables qui se spécialisent dans ce genre d'escroquerie. Inutile d'insister pour démontrer que les «United Electrical, Radio and Machine Workers of America » étaient opposés au partage des profits.

(7) Senate Report, op. cit., p. 107.

(8) Of. Congressional Digest, p. 24.

(9) Cf. Morris L. Cooke et Philip Murray, Organized Labor and Production, p. 121.

(10) Cf. Robert L. Dixon, Profit Sharing, The Journal of Accountancy, July 1946. 
Lors de la fondation du "Council of Profit Sharing Industries》, en 1947, le docteur Robert Hartman signalait que le mouvement ouvrier, comme tel, n'était pas représenté à cette assemblée. Il s'agit, disait-il, d'une assemblée de patrons. William Green, président de la Fédération américaine du travail, avait auparavant adressé une lettre au docteur Hartman pour l'informer qu'il y avait des divergences d'opinion au sein de la Fédération américaine du travail au sujet du mérite, des principes et des buts du système de partage des profits. ${ }^{11}$ Green terminait par une invitation à $\mathrm{M}$. Hartman d'aller le rencontrer à Washington pour discuter de l'affaire avec lui.

Quant au Congrès des organisations industrielles, il adressa aussi a M. Hartman une lettre signée par Kermit Eby, dans laquelle on soulignait que cette centrale ouvrière ne pouvait pas, à ce moment, prendre position. ${ }^{12} \mathrm{Au}$ cours des années suivantes, le «Council of Profit Sharing Industries » a réussi à attirer des représentants ouvriers à ses congrès, mais dans chaque cas il s'agissait d'officiers locaux ou de personnes préposées à la recherche et à l'éducation.

Les raisons de l'indifférence, de la réticence ou de l'opposition du mouvement ouvrier sont assez nombreuses. La participation aux profits apparaît aux travailleurs comme un truc pour éviter le paiement dé bons salaires. Comme on l'a vu aussi, les dirigeants ouvriers considèrent le mouvement de partage des profits comme une affaire patronale, conçue, appliquée et contrôlée par les employeurs. La participation aux profits a souvent été aussi le privilège exclusif d'un groupe restreint de hauts salariés dans une entreprise, ce qui, dans ces cas, ne l'a pas rendue très populaire. L'une des autres objections des ouvriers, on l'a vu déjà, porte sur les méthodes comptables de détermiuation des profits, méthodes mal comprises des travailleurs et sur les. quelles ils n'ont aucun contrôle. Le fait que les ouvriers n'ont aucune influence directe sur la marche de l'entreprise, notamment sur sa politique commerciale, n'est pas de nature à leur faire accepter avec enthousiasme qu'une partie importante de leur revenu dépende du volume des profits, d'autant plus que cette source de rémunération est aléatoire et variable. L'une des plus vieilles objections du mouvement ouvrier à la participation aux bénéfices provient de ce que cette formule a souvent été considérée comme une forme subtile de «speed-up » ou d'acoélération indue du rythme de travail. De plus, la participation

(11) Cf. Proceedings, Founding Conference, op. cit., p. 27.

(12) Op. cit., p. 27. 
aux profits a pour effet d'identifier en quelque sorte l'intérêt de l'ouvrier avec celui du patron, à le faire embarquer dans la même galère, à l'émanciper par miettes sur le plan de l'entreprise en le détachant de l'ensemble du mouvement ouvrier. Cette participation pose ainsi au travailleu:, dans bien des cas, un conflit d'allégeance. Enfin, le mouvement ouvrier dans son ensemble a boudé ou combattu la participation aux profits parce que cette technique a souvent été utilisée pour combattre l'organisation ouvrière, pour diminuer le prestige des syndicats auprès de ses membres, pour éviter la négociation de conventions collectives ou pour en diminuer l'importance.

\section{Le rôle des travailleurs dans la participation aux bénéfices}

Bien que la politique officielle du mouvement ouvrier américain n'ait jamais été favorable à la participation aux bénéfices, il serait faux de conclure que le rôle des travailleurs y soit entièrement passif. Il existe de nombreux cas particuliers de collaboration efficace entre employeurs et unions locales ou même nationales dans ce domaine.

Dans certains cas, des plans de participation aux bénéfices ont été institués à la demande d'une union ouvrière; dans d'autres cas, il est arrivé que l'union a formé un comité pour étudier la question et pour mettre au point avec l'employeur un plan satisfaisant pour les deux parties. ${ }^{13}$ En 1951, une union internationale, la «International Union of Electrical Workers », fit une demande officielle de participation aux bénéfices dans ses négociations avec les compagnies «General Electric》 ct «Westinghouse ». ${ }^{14}$ Les compagnies répliquèrent que cette question ne relevait pas de la négociation de conventions collectives. Ce qui est significatif, ici, c'est que pour la première fois une importante union ouvrière prenait une telle initiative.

Dans une allocution prononcée devant le «Council of Profit Sharing Industries » en décembre 1949, Georges Baldanzi, vice-président de la «Textile Workers Union», se déclarait en faveur de la participation aux bénéfices à condition que celle-ci soit bien appliquée. ${ }^{15} \mathrm{M}$. Cass D. Alvin, en charge de la publicité et de l'éducation pour les «United Steel Workers of America », signalait au congrès de 1955 du

(13) Cf. National Industrial Conference Board, Profit Sharing for Workers, Studies in Personnel Policy no. 97, 1948, p. 29.

(14) Cf. Flippo, op. cit., p. 90.

(15) Cf. Business Week, "Labor Man for Profit Sharing", 10 déc. 1949, pp. 56-57. 
«Council of Profit Sharing Industries» que plusieurs compagnies avec lesquelles son union négociait avaient des plans de participation aux bénéfices. Et il ajoutait que l'un des auteurs les plus connus sur la participation aux profits n'était nul autre que Joseph N. Scanlon, un ancien officier de cette union ouvrière et un conseiller intime du président de l'union, David J. McDonald. ${ }^{16}$ En fait, les «United Steel Workers of America » ont participé largement à l'élaboration d'un plan de partage des profits à la "Adamson Company », Ohio. ${ }^{17}$ En 1946, Eric Johnston instituait la participation aux bénéfices dans ses quatre établissements de la Côte du Pacifique. Dans ces quatre établissements, la compagnie avait signé des conventions collectives avec des unions affiliées à la Fédération américaine du travail. ${ }^{18}$

Dès 1938, le président du «National Council of Independent Uinions» avait déclaré devant le sous-comité sénatorial que l'idée de participation aux bénéfices lui paraissait bonne en principe. ${ }^{19}$ Il reste que ces exemples d'attitudes syndicales en faveur de la participation aux bénéfices sont des phénomènes isolés et qu'il serait osé de prétendre y déceler une nouvelle tendance. Malgré tout, ils indiquent peutêtre un léger fléchissement dans l'opposition du syndicalisme.

Des enquêtes intéressantes ont tenté, surtout au cours des dernières années, de mesurer objectivement le degré de la participation ouvrière dans le mouvement de partage des profits. L'une des plus complètes est celle de Flippo. ${ }^{20}$ Les résultats de cette étude proviennent de réponses fournies par 350 compagnies sur les 848 à qui l'auteur avait fait parvenir un questionnaire. Sur 341 de ces compagnies, il y en avait 147 , soit $43.1 \%$, qui traitaient avec des unions, dont 51 affiliées à la Fédération américaine du travail et 46 au Congrès des organisations industrielles. ${ }^{21}$ Pour ce groupe de compagnies, Flippo signale l'existence de 129 conventions collectives. Ce qui est significatif, c'est que 96 de ces 129 conventions collectives ne contiennent aucune disposition relative au partage des profits. Dans 15 seulement des 129 conventions, le plan de partage est inclus et détaillé; dans 18 autres, on ny trouve qu'une allusion ou une brève référence. Ainsi, c'est dans 15 cas seulement sur les 3.50 couverts par l'enquête, c'est-à-dire dans une

(16) The 1955 Transcript, op. cit., p. 87.

(17) Cf. Thompson, op. cit., D. 54.

(18) Cf. Business Week, "Profits Shared", 26 janv. 1946, p. 97.

(19) Senate Report, op. cit., p. 92.

(20) Op. cit.

(21) Ibid., p. 93. 
proportion de $4.2 \%$, que le plan de partage des bénéfices résulte d'un accord explicite entre un employeur et une union ouvrière. Dans les 33 cas où l'on trouve des dispositions sur le partage des profits dans les conventions, il semble y avoir une tendance évidente en faveur des formules comportant une distribution immédiate. ${ }^{22}$ Cette constatation semble indiquer une préférence des unions ouvrières concernées pour des formules qui ressemblent plus au régime du salariat que les plans à distribution différée, surtout lorsque ces plans comportent une contribution de l'ouvrier à un fonds de fiducie.

Sur 129 expériences étudiées en 1945 par les «Industrial Relations Counselors », on constate que dans 117 cas l'administration du plan est l'affaire exclusive du bureau de direction de la compagnie. ${ }^{23}$

Dans la plupart des cas étudiés lors d'une enquête sur la région cie Chicago, ${ }^{24}$ les employés ne participent aucunement à l'administration du plan de partage des profits. Quelques-unes de ces compagnies ne donnent aux employés aucun renseignement sur leur situation financière ni sur l'état du fonds de fiducie alimenté par les contribuuons patronales. ${ }^{25}$ De plus, il n'existe aucune convention collective avec des unions ouvrières dans ces industries. ${ }^{26}$

Le «Council of Profit Sharing Industries», pour sa part, signale que près de $40 \%$ de ses membres comptent des unions ouvrières. ${ }^{2 \tau}$

Dans l'enquête de Knowlton, 91 des 300 compagnies qui ont répondu à son questionnaire indiquent que leurs ouvriers sont syndiqués dans une proportion de $40 \%$ ou plus; par contre, dans 166 cas, les ouvriers ne sont pas syndiqués ou ne le sont que dans une proportion de moins de $10 \%$; dans 20 cas, la proportion des ouvriers syndiqués varie entre $10 \%$ et $40 \% .{ }^{28}$

Une excellente étude sur l'utilisation et l'administration des fonds de fiducie alimentés par des contributions patronales prises à même les profits a été faite par J.J. Jehring, directeur de la «Profit Sharing Re-

(22) Cf. Flippo, op. cit., p. 99.

(23) Stewart et Couper, op. cit., p. 43.

(24) Il s'agit d'une enquête faite par Kenneth M. Thompson, auteur de "Profit Sharing Democratic Capitalism..." (op. cit.).

(25) Cf. Thompson, op. cit., p. 44.

(26) Idem, v. 49.

(27) Cf. Revised Profit Sharing Manual, op. cit., p. 3.

(28) Cf. Knowlton, op. cit., p. 10. 
search Foundation ». Il s'agit d'une enquête faite auprès de 700 compagnies. ${ }^{29} 208$ d'entre elles ont rempli le questionnaire qui leur avait été adressé. Ce sont les réponses de ces compagnies que le livre de Jehring présente, analyse et commente.

Voici quelques-uns des principaux faits révélés par l'enquête. Sur 11 ccmpagnies employant 5,000 ouvriers et plus, 5 indiquent qu'il existe un comité consultatif comprenant des représentants ouvriers; ces 5 compagnies ont affaire à des unions ouvrières, mais aucune de ces dernières n€ participe de quelque façon que ce soit à l'administration des fonds. ${ }^{3 \circ}$

Sur 26 compagnies employant de 1,500 à 5,000 ouvriers, 13 indiquent qu'il existe un comité consultatif comprenant des représentant3 ouvriers; 13 compagnies signalent qu'elles ont affaire à des unions ouvrières, mais dans 2 cas seulement ces unions ont des représentants au sein du comité qui participent à l'administration du fonds. ${ }^{31}$

Sur 44 compagnies employant de 500 à 1,500 ouvriers, 29 indiquent l'existence d'un comité consultatif comprenant des représentants des employés; 17 avaient affaire à des unions ouvrières, mais dans 6 cas seulement l'union participait à l'administration du fonds. ${ }^{32}$

Sur 64 compagnies employant de 100 à 500 personnes, 44 indiquent l'existence d'un comité consultatif comprenant des représentants ouvriers; 23 avaient affaire à des unions, mais dans 3 cas seulement l'union participait à l'administration du fonds.

Dans le groupe des 36 compagnies employant 100 ouvriers ou moins, 26 indiquent qu'il existe des comités consultatifs comprenant des représentants ouvriers; 9 d'entre elles ont affaire à des unions, mais dans 2 cas seulement l'union participe à l'administration du fonds.

A la fin de son volume, Jehring fait une récapitulation des résultats en tenant compte de toutes les catégories d'entreprises étudiées. Dans $63.5 \%$ des cas, on constate l'existence d'un comité consultatif comprenant des représentants des employés. Ces comités sont plus nombreux dans les plus petites compagnies $(71 \%)$ que dans les plus grandes $(36 \%)$, et le nombre de membres augmente avec la dimension de l'en-

(29) J.J. Jehring, The Investment and Administration..., op. cit., p. 5.

(30) J.J. Jehring, op. cit., p. 9.

(31) Ibid., D. 20.

(32) Ibid., D. 34. 
treprise. Dans la grande majorité des cas, les membres de ces comités sont désignés par les administrateurs de l'entreprise ou le bureau de direction. Parfois, ils sont élus par les employés. La plupart des employés qui siègent à ces comités se recrutent parmi le personnel administratif. Dans certains cas, des travailleurs d'usine en font partie. ${ }^{33}$

Dans sa récapitulation couvrant toutes les catégories d'entreprises, Jehring signale encore que même si $37 \%$ des compagnies ont affaire à une ou à des unions ouvrières, l'union ne participe à l'administration des fonds que dans $6 \%$ seulement des cas. ${ }^{34}$ L'auteur ajoute qu'il n'a constaté de lien entre le plan de partage des profits et la comvention collective que dans un très petit nombre de cas. ${ }^{35}$

Après l'examen objectif de tous les faits rapportés précédemment, il faut tirer la conclusion que les ouvriers, individuellement ou collectivement, ne sont pas des agents importants dans le mouvement de participation aux bénéfices. Ils n'ont pas pris part à l'élaboration de son idéologie, ils ne sont que très rarement les initiateurs de l'expérience dans une entreprise, ils ne donnent pas souvent leur accord collectif aux plans qui entrent en vigueur sous l'initiative du patron, enfin leur influence sur l'utilisation des sommes provenant des contributions patronales et des leurs à un fonds de fiducie est presque nulle.

Four ces raisons, il est clair que le mouvement de participation aux bénéfices n'a pas encore réussi, malheureusement, à atteindre l'un de ses principaux objectifs humains, c'est-à-dire la promotion ouvrière par l'intégration progressive des travailleurs dans la vie de l'entreprise. Le pire, c'est qu'il ne semble pas y avoir d'indice factuel sérieux d'une tendance dans cette direction.

\section{Labor Attitudes on Profit Sharing in the United States}

Profit sharing is prominently a management and governmental affair. Labor as a whole has not taken a very active part in the development of this idea and practice.

Labor attitudes can be studied three different levels: 1) the workers in a particular firm or as individuals spread across the country; 2) as local union officers; 3) as national or international union officers. These attitudes can also be considered with an historical approach.

(33) Jehrung, op. cit., p. 93. (34) Jehrung, op. cit., p. $94 . \quad$ (35) Ibid., p. 94. 
As far as workers in a firm or as individuals in the total labor force of the country are concerned, the Senate sub-committee showed in 1938 that most of them were in favor of profit sharing. As a matter of fact, $87 \%$ of those who gave an answer on this question were favorable to this idea. Other surveys seem to confirm this finding, although no systematic investigations have been carried out in labor circles on this particular point.

Local union officers have a mixed feeling on profit sharing. Sometimes they are inclined to take favorable action on it because of their membership desires, but they are hesitant about it because it would run across a national policy of their union or even only hurt the prevailing ideas in national circles. Usually, however, where membership and company pressures are strong, the local union officers will readily cooperate.

Generally speaking, national labor leaders have been systematically indifferent or opposed to profit sharing. Samuel Gompers, first president of the American Federation of Labor, John L. Lewis, William Green have all emphatically stated their opposition to profit sharing before the Senate sub-committee in 1938 . Of these three leaders, Green is the only one who gave an indication that his attitude was not due to the principles involved, but rather to their actual applications. Philip Murray, when he was president of the Congress of Industrial Organizations, felt that profit sharing was a cause of dispute rather than a means of achieving peace in industrial relations.

Labor has many reasons for its opposition to profit sharing. This practice is considered by many workers as a device to escape the payment of fair wages and as a method to cheat them on accounting techniques. They do not like to see part of their pay subject to the fluctuations of a profit on which they have no direct or immediate control. They figure in many cases that profit sharing is just another form of speed-up. In some cases also, labor men feel that profit sharing requires a shift of loyalty from the labor movement towards a particular company. Generally, labor has been opposed to profit sharing because this idea has been used very often to fight unionism and collective bargaining.

Although the general picture shows an unfavorable attitude in labor circles, one can still find a great number of particular instances - some of which are of great importance - where management and union officials have worked out together successful profit sharing plans. In some cases, big national unions have made a formal request for profit sharing in their negotiations with companies.

Nevertheless, it is a fact that unions very seldom have anything to do in a profit sharing plan. While any survey may show that profit sharing plans are operating in a great number of plants where a union exists, the plan is rarely spelled out or even mentioned in the collective labor agreement.

As far as administration of profit sharing funds is concerned, labor has nothing to do in almost all cases. In the few instances where the union has something to say in this administration, management usually still has the last word.

The conclusion is that profit sharing, unfortunately, has not suceeded, yet, in promoting labor in the way of an actual sharing of responsibilities with management. There seems to be no existing indication of any slight trend in that direction. 